\title{
Open k-monopolies in graphs: complexity and related concepts
}

\author{
Dorota Kuziak $^{1} \quad$ Iztok Peterin ${ }^{2,3 *} \quad$ Ismael G. Yero ${ }^{4}$ \\ 1 Departament d'Enginyeria Informàtica i Matemàtiques, Universitat Rovira i Virgili, Spain \\ 2 University of Maribor, Faculty of Electrical Engineering and Computer Science, Slovenia \\ 3 Institute of Mathematics, Physics and Mechanics, Slovenia \\ 4 Escuela Politécnica Superior, Universidad de Cádiz, Spain
}

received $12^{\text {th }}$ June 2015, revised $17^{\text {th }}$ Nov. 2015, accepted $7^{\text {th }}$ Mar. 2016.

Closed monopolies in graphs have a quite long range of applications in several problems related to overcoming failures, since they frequently have some common approaches around the notion of majorities, for instance to consensus problems, diagnosis problems or voting systems. We introduce here open $k$-monopolies in graphs which are closely related to different parameters in graphs. Given a graph $G=(V, E)$ and $X \subseteq V$, if $\delta_{X}(v)$ is the number of neighbors $v$ has in $X, k$ is an integer and $t$ is a positive integer, then we establish in this article a connection between the following three concepts:

- Given a nonempty set $M \subseteq V$ a vertex $v$ of $G$ is said to be $k$-controlled by $M$ if $\delta_{M}(v) \geq \frac{\delta_{V}(v)}{2}+k$. The set $M$ is called an open $k$-monopoly for $G$ if it $k$-controls every vertex $v$ of $G$.

- A function $f: V \rightarrow\{-1,1\}$ is called a signed total $t$-dominating function for $G$ if $f(N(v))=\sum_{v \in N(v)} f(v) \geq$ $t$ for all $v \in V$.

- A nonempty set $S \subseteq V$ is a global (defensive and offensive) $k$-alliance in $G$ if $\delta_{S}(v) \geq \delta_{V-S}(v)+k$ holds for every $v \in V$.

In this article we prove that the problem of computing the minimum cardinality of an open 0-monopoly in a graph is NP-complete even restricted to bipartite or chordal graphs. In addition we present some general bounds for the minimum cardinality of open $k$-monopolies and we derive some exact values.

Keywords: open $k$-monopolies, $k$-signed total domination, global defensive $k$-alliance, global offensive $k$-alliance

\section{Introduction}

We begin stating some terminology and notation which we will use. Throughout this article, $G$ denotes a simple graph with vertex set $V(G)$ and edge set $E(G)$ (we will use only $V$ and $E$ if the graph is clear from the context). The order of $G$ is $n=|V(G)|$ and the size is $m=|E(G)|$. We denote two adjacent vertices $u$ and $v$ by $u \sim v$. Given a vertex $v \in V$, the set $N(v)=\{u \in V: u \sim v\}$ is the open neighborhood of

* Partially supported by the Ministry of Science of Slovenia under the grant P1-0297.

ISSN 1365-8050 C 2016 by the author(s) Distributed under a Creative Commons Attribution 4.0 International License 
$v$, and the set $N[v]=N(v) \cup\{v\}$ is the closed neighborhood of $v$. So, the degree of a vertex $v \in V$ is $\delta(v)=|N(v)|$. Given a set $S \subset V$, the open neighborhood of $S$ is $N(S)=\bigcup_{v \in S} N(v)$ and the closed neighborhood of $S$ is $N[S]=N(S) \cup S$. The minimum and maximum degree of $G$ are denoted by $\delta(G)$ and $\Delta(G)$, respectively (again we use $\delta$ and $\Delta$ for short if $G$ is clear from the context). For a nonempty set $S \subseteq V$ and a vertex $v \in V, N_{S}(v)$ denotes the set of neighbors $v$ has in $S$, i.e., $N_{S}(v)=S \cap N(v)$. The degree of $v$ in $S$ will be denoted by $\delta_{S}(v)=\left|N_{S}(v)\right|$. Also, $\bar{S}=V-S$ is the complement of a set $S$ in $V$ and $\partial S=N[S]-S$ is the boundary of a set $S$. The subgraph of $G$ induced by a set $S$ is denoted by $\langle S\rangle$.

In the first article, see Linial et al. (1993), on closed monopolies in graphs (called monopolies there) the following terminology was used. A vertex $v$ in $G$ is said to be controlled by a set $M \subset V$ if at least half of its closed neighborhood is in $M$. The set $M$ is called a closed monopoly if it controls every vertex $v$ of $G$. Equivalently, the set $M$ is a closed monopoly in $G$, if for any vertex $v \in V(G)$ it follows that $|N[v] \cap M| \geq\left[\frac{|N[v]|}{2}\right]$. In this article, we introduce open $k$-monopolies in a natural way, by replacing closed neighborhoods with open neighborhoods. Hence, we can use the degree of vertices instead of cardinalities of closed neighborhoods. Given some integer $k$, a vertex $v$ of $G$ is said to be $k$-controlled by a set $M$ if $\delta_{M}(v) \geq \frac{\delta(v)}{2}+k$. Analogously, the set $M$ is called an open $k$-monopoly if it $k$-controls every vertex $v$ of $G$. Notice that not for every value of $k$ there exists an open $k$-monopoly in $G$ (further on we give some suitable interval for such $k$ ). Also, note that, close and open monopolies cannot be exactly compared, since in a closed monopoly a vertex $v$ also counts itself in controlling $v$, which is not the case in any open monopoly. The smallest example is already $K_{2}$, where is only one vertex in a closed monopoly, but both vertices are necessary in an open 0-monopoly. Differently, there are only two vertices in a minimum open 0 -monopoly of $P_{5}$, while we need at least three vertices in every closed monopoly of $P_{5}$. In this article, we are focused only in open $k$-monopolies. In this sense, from now on we omit the term "open" and just use the terminology of $k$-monopolies. On the other hand, we remain using the term closed monopoly whenever referring to some previous work on this topic.

According to Bermond et al. (2003), several problems related to overcoming failures have some common approaches around the notion of majorities. Their ideas are directed toward decreasing, as much as possible, the damage caused due to failed vertices; by maintaining copies of the most important data and performing a voting process among the participating processors in situation that failures occur; and by adopting as true those data stored at the majority of the not failed processors. This idea is also commonly used in some fault tolerant algorithms including agreement and consensus problems (see Dwork et al. (1988)), diagnosis problems (see Sullivan (1986)) or voting systems (see Garcia-Molina and Barbara (1985)), among other applications and references.

Bermond et al. (2003) were interested into locality based on the following facts. Frequently, processors running in a system are better aware of whatever happens in their neighborhood than outside of it. Moreover, some distributed network models allow only for computations developed with local processors, which means that, a processor can only obtain a data from other processors having a "relative" close distance from itself. Therefore, it is more efficient to store data as locally as possible.

Nevertheless, there could exists also a risk in this way. If the voting is restricted to local neighborhoods, we could produce a sufficiently large set of failures which will probably constitute the majority in some of these neighborhoods. In this sense, see Bermond et al. (2003), the authors assert the following: once the voting is performed over subsets of vertices, the ability of failed vertices to influence the outcome of the votes becomes not only a function of their number but also a function of their location in the network: 
well situated vertices can acquire greater influence. This simple fact led them to study the problem of characterizing the potential power of a set of failures in a network of processors, and as a consequence, the study of (closed) monopolies in graphs.

Notions of closed monopolies in graphs were introduced first by Linial et al. (1993), where several ideas regarding voting systems were described. Once such article appeared, a high number of researches were devoted to such parameter and its relationship with other similar structures like (defensive and offensive) alliances, see Kristiansen et al. (2004), or signed dominating functions, see Dunbar et al. (1995), among other works. An interesting article, where several of these connections are dealt with, is from Fernau and Rodríguez-Velázquez (2014). Moreover, this article presents a possible generalization of all these (closed) monopolies-related structures which comprise them altogether. The complexity of closed monopolies in graphs is also well studied. The NP-hardness of finding the minimum cardinality of a closed monopoly in a graph is easy to observe as stated by Linial et al. (1993). In such work was also pointed out a conjecture concerning the inapproximability of such problem. A weaker version of such conjecture has been proved by Mishra et al. (2002). In addition some other inapproximability results of this problem have appeared by Mishra (2012) and Mishra and Rao (2006). Particularly, in Mishra and Rao (2006), these results are centered in regular graphs. Moreover, there it is also proved that for the case of tree graphs, a closed monopoly of minimum cardinality can be computed in linear time. On the other hand, see Khoshkhah et al. (2013), some relationships and bounds for the minimum cardinality of closed monopolies in graphs are stated in terms of matchings and/or girths. Also, dynamic closed monopolies has been introduced in connection with modeling some problems of spreading the influence in social networks (see Bermond et al. (2003); Peleg (2002)). Other studies in dynamic closed monopolies can be found in Flocchini et al. (2003) and in Zaker (2012).

\section{Concepts related to monopolies}

Many times mathematical concepts are defined independently in two or even more papers. When this occurs, the equivalence sometimes is obvious (mostly when papers occur in the same time period), but sometimes we need more effort to find the connection (mostly when there is a longer time period between publications). This may yield not sufficient effort of later authors with the history, but we rather present it as an enough important concept to start to investigate it from different point of view.

The above holds (at least partial) for signed (total) domination (introduced first by Hattingh et al. (1995) (by Zelinka (2001))) and for different types of alliances (introduced first by Kristiansen et al. (2004)). We add monopolies to this list and present these connections in this section.

\subsection{Alliances}

Alliances in graphs were introduced first by Kristiansen et al. (2004) and generalized to $k$-alliances by Shafique and Dutton $(2003,2006)$. After that several works have been developed in this topic. Remarkable cases are Favaron et al. (2004) and Haynes et al. (2003). Relationships with different parameters of the graphs have been obtained and the alliances of several families of graphs have been studied. A nonempty set $S \subseteq V$ is a defensive $k$-alliance in $G$ for $k \in\{-\Delta, \ldots, \Delta\}$ if for every $v \in S$

$$
\delta_{S}(v) \geq \delta_{\bar{S}}(v)+k .
$$


Moreover, for $k \in\{2-\Delta, \ldots, \Delta\}$, a nonempty set $S \subseteq V$ is an offensive $k$-alliance in $G$ if for every $v \in \partial S$

$$
\delta_{S}(v) \geq \delta_{\bar{S}}(v)+k .
$$

A nonempty set $S \subseteq V$ is a powerful $k$-alliance if $S$ is a defensive $k$-alliance and an offensive $(k+2)$ alliance. A set $D$ is a dominating set in $G$ if every vertex outside of $D$ is adjacent to at least one vertex of $G$.

A (defensive, offensive or powerful) $k$-alliance is called global if it is a dominating set. The global defensive (offensive) $k$-alliance number of $G$, denoted by $\gamma_{k}^{d}(G)\left(\gamma_{k}^{o}(G)\right.$ ), is defined as the minimum cardinality of a global defensive (offensive) $k$-alliance in $G$. For $k \in\{-\Delta, \ldots, \Delta-2\}$, the global powerful $k$-alliance number of $G$, denoted by $\gamma_{k}^{p}(G)$, is defined as the minimum cardinality of a global powerful $k$-alliance in $G$. A global powerful alliance of minimum cardinality in $G$ is called a $\gamma_{k}^{p}(G)$-set of $G$. Notice that there exist graphs not containing any global powerful $k$-alliance for some specific values of $k$. In this sense, in this work we are interested in those graphs having global powerful $k$-alliances. It means that whenever we study such an alliances we are supposing that the graph contains it.

Notice that the terminology used for alliances provides a very useful tool which can be used while proving several results, i.e., a set of vertices $M$ is a $k$-monopoly in $G$ if and only if for every vertex $v$ of $G, \delta_{M}(v) \geq \delta_{\bar{M}}(v)+2 k$ (from now we will call this expression the $k$-monopoly condition) and we will say that $M$ is a $k$-monopoly in $G$ if and only if every $v$ of $G$ satisfies the $k$-monopoly condition for $M$.

An interesting possible generalization of alliances in graphs (and some other related parameters) is given by Fernau and Rodríguez-Velázquez (2014). In this work is proposed a new framework, which the authors call $(D, O)$-alliances. The main idea of this allows not only to characterize several known variants of alliances, but also suggest a unifying framework for its study. In this sense, a $(D, O)$-alliance, with $D, O \subseteq \mathbb{Z}$ in a graph $G=(V, E)$ is a set $S$ such that for any $v \in S, \delta_{S}(v)-\delta_{\bar{S}}(v) \in D$ and for any $v \in N(S) \backslash S, \delta_{S}(v)-\delta_{\bar{S}}(v) \in O$. According to this, it is clear to observe that a defensive $k$-alliance can be understood as a $(\{z \in \mathbb{Z}: z \geq k\}, \mathbb{Z})$-alliance, and an offensive $k$-alliance as a $(\mathbb{Z},\{z \in \mathbb{Z}: z \geq k\})$-alliance.

\subsection{Signed (total) domination}

Given a graph $G=(V, E)$ and a function $f: V \rightarrow\{-1,1\}$ we consider the following for $f$ :

- $f$ is a signed dominating function for $G$ if $f(N[v])=\sum_{u \in N[v]} f(u) \geq 1$, for all $v \in V$.

- $f$ is a signed total dominating function for $G$ if $f(N(v))=\sum_{u \in N(v)} f(u) \geq 1$, for all $v \in V$.

- $f$ is a signed $k$-dominating function for $G$ if $f(N[v]) \geq k$ for all $v \in V$.

- $f$ is a signed total $k$-dominating function for $G$ if $f(N(v)) \geq k$ for all $v \in V$.

The minimum weight $\sum_{v \in V} f(v)$ of a signed (total) ( $k$-dominating) dominating function $f$ is the signed (total) ( $k$-domination) number of $G$ and they are denoted in the following way.

\begin{tabular}{|c|c|c|c|}
\hline signed domination & signed total domination & signed $k$-domination & signed total $k$-domination \\
\hline$\gamma_{s}(G)$ & $\gamma_{s t}(G)$ & $\gamma_{s}^{k}(G)$ & $\gamma_{s t}^{k}(G)$ \\
\hline
\end{tabular}

Notice that, if $k=1$, then a signed (total) 1-dominating function is a standard signed (total) dominating function for $G$. Also, any kind of signed (total) ( $k$-dominating) dominating function $f$ of $G$ induces two 
disjoint sets of vertices $B_{1}$ and $B_{-1}$, such that for every vertex $v \in B_{i}, f(v)=i$ with $i \in\{-1,1\}$. Hereby we will represent such a function $f$ by the sets $B_{1}$ and $B_{-1}$ induced by $f$ and we will write $f=\left(B_{1}, B_{-1}\right)$. A signed (total) ( $k$-dominating) dominating function $f$ of minimum weight is called a $\gamma$-function with $\gamma \in\left\{\gamma_{s}(G), \gamma_{s t}(G), \gamma_{s}^{k}(G), \gamma_{s t}^{k}(G)\right\}$, respectively.

\subsection{Connections between concepts}

Observing the definitions of monopoly and alliance we see that both concepts are closely related. That is, let $M$ be a 0 -monopoly in $G=(V, E)$ and let $v \in V$. Hence, $v$ has at least half of its neighbors in $M$, i.e., $\delta_{M}(v) \geq \frac{\delta(v)}{2}$, which leads to $\delta_{M}(v) \geq \delta_{\bar{M}}(v)$. Since this is satisfied for every vertex of $G$ we obtain that $M$ is a global defensive 0 -alliance and also a global offensive 0 -alliance. On the contrary, let $A$ be a global defensive 0 -alliance which is also a global offensive 0 -alliance in $G$. Hence, for every vertex $u \in V$ we have that $\delta_{A}(v) \geq \delta_{\bar{A}}(v)$, which leads to $\delta_{A}(v) \geq \frac{\delta(v)}{2}$. Therefore, $A$ is a 0 -monopoly.

Shafique and Dutton (2003) defined the concept of global powerful $k$-alliances. Nevertheless, it was not taken into account the possibility of studying the cases in which a set is a global defensive $k$-alliance and also a global offensive $k$-alliance. According to the concept of monopoly we observe the importance of such a case, which is one of our motivations to develop the present investigation.

We continue with a relationship between signed total domination, alliances and monopolies.

Theorem 1. Let $G=(V, E)$ be a graph and let $k \in\left\{1, \ldots,\left\lfloor\frac{\delta(G)}{2}\right\rfloor\right\}$ be an integer. The following statements are equivalent:

(i) $M \subset V$ is a k-monopoly in $G$;

(ii) $M$ is a global defensive (2k)-alliance and a global offensive (2k)-alliance in $G$;

(iii) $f=\left(B_{1}=M, B_{-1}=\bar{M}\right)$ is a signed total ( $\left.2 k\right)$-dominating function for $G$.

Moreover, if $k=0$, then (i) and (ii) are also equivalent.

Proof: The equivalence between (i) and (ii) is straightforward since for every set of vertices $M$ and every vertex $v$ of $G$, the conditions $\delta_{M}(v) \geq \frac{\delta(v)}{2}+k$ and $\delta_{M}(v) \geq \delta_{\bar{M}}(v)+2 k$ are equivalent for every $k \in\left\{0, \ldots,\left\lfloor\frac{\delta(G)}{2}\right\rfloor\right\}$.

Let $M$ be a global defensive $(2 k)$-alliance and a global offensive $(2 k)$-alliance in $G$. Let the function $f: V \rightarrow\{-1,1\}$ be such that for any $v \in V$, it follows $f(v)=1$ if $v \in M$ and, $f(v)=-1$ otherwise. If $v \in M$, then since $M$ is a global defensive $(2 k)$-alliance in $G$, we have that

$$
\begin{aligned}
f(N(v)) & =f\left(N_{M}(v)\right)+f\left(N_{\bar{M}}(v)\right) \\
& =\delta_{M}(v)-\delta_{\bar{M}}(v) \\
& \geq \delta_{\bar{M}}(v)+2 k-\delta_{\bar{M}}(v) \\
& =2 k
\end{aligned}
$$

Now, if $v \in \bar{M}$, then by using that $M$ is a global offensive $(2 k)$-alliance in $G$, the same computation as above gives that $f=\left(B_{1}=M, B_{-1}=\bar{M}\right)$ is a signed total $(2 k)$-dominating function for $G$. 
On the other hand, let $f^{\prime}=\left(B_{1}^{\prime}, B_{-1}^{\prime}\right)$ be a signed total $(2 k)$-dominating function for $G$. Let $M^{\prime}=B_{1}^{\prime}$ and let the vertex $u \in V$. If $u \in M^{\prime}$, then since $f^{\prime}$ is a signed total $(2 k)$-dominating function in $G$, we have that

$$
\begin{aligned}
\delta_{M^{\prime}}(u) & =f^{\prime}\left(N_{M^{\prime}}(u)\right) \\
& =f^{\prime}(N(u))-f^{\prime}\left(N_{\overline{M^{\prime}}}(u)\right) \\
& \geq 2 k-f^{\prime}\left(N_{\overline{M^{\prime}}}(u)\right) \\
& =\delta_{\overline{M^{\prime}}}(u)+2 k .
\end{aligned}
$$

Thus, $M^{\prime}$ is a global defensive $(2 k)$-alliance in $G$. Finally, since $f^{\prime}$ is a signed total $(2 k)$-dominating function for $G$, if $u \in \overline{M^{\prime}}$, then as above we deduce that $M^{\prime}$ is a global offensive $(2 k)$-alliance.

The following corollary is a direct consequence of Theorem 1 (ii) and (iii). We omit the proof.

Corollary 2. Let $G=(V, E)$ be a graph and let $k \in\left\{1, \ldots,\left\lfloor\frac{\delta(G)}{2}\right\rfloor\right\}$ be an integer. A set $M \subset V$ is a global defensive $k$-alliance and a global offensive $k$-alliance in $G$ if and only if $f=\left(B_{1}=M, B_{-1}=\right.$ $\bar{M})$ is a signed total $k$-dominating function for $G$.

Now we prove a connection between signed domination and powerful alliances.

Theorem 3. Let $G=(V, E)$ be a graph and let $k \in\{0, \ldots, \delta(G)\}$. Then $S \subset V$ is a global powerful $k$-alliance in $G$ if and only if $f=\left(B_{1}=S, B_{-1}=\bar{S}\right)$ is a signed $(k+1)$-dominating function for $G$.

Proof: Let $S$ be a global powerful $k$-alliance in $G$. So, $S$ is a global defensive $k$-alliance and a global offensive $(k+2)$-alliance in $G$. Let $f=\left(B_{1}=S, B_{-1}=\bar{S}\right)$ be a function in $G$ and let $v \in V$. We consider the following cases.

Case 1: $v \in S$. Since $S$ is a global defensive $k$-alliance in $G$, we have that

$$
\begin{aligned}
f(N[v]) & =f\left(N_{S}(v)\right)+f\left(N_{\bar{S}}(v)\right)+1 \\
& =\delta_{S}(v)-\delta_{\bar{S}}(v)+1 \\
& \geq \delta_{\bar{S}}(v)+k-\delta_{\bar{S}}(v)+1 \\
& =k+1 .
\end{aligned}
$$

Case 2: $v \in \bar{S}$. Since $S$ is a global offensive $(k+2)$-alliance in $G$, we have that

$$
\begin{aligned}
f(N[v]) & =f\left(N_{S}(v)\right)+f\left(N_{\bar{S}}(v)\right)-1 \\
& =\delta_{S}(v)-\delta_{\bar{S}}(v)-1 \\
& \geq \delta_{\bar{S}}(v)+k+2-\delta_{\bar{S}}(v)-1 \\
& =k+1 .
\end{aligned}
$$

Thus, $f=\left(B_{1}=S, B_{-1}=\bar{S}\right)$ is a signed $(k+1)$-dominating function for $G$.

On the other hand, let $f^{\prime}=\left(B_{1}^{\prime}, B_{-1}^{\prime}\right)$ be a signed $(k+1)$-dominating function in $G$. We will show that $A=B_{1}^{\prime}$ is a global powerful $k$-alliance in $G$. Let $u \in V$. We consider the following. 
Case 3: $u \in A$. Since $f^{\prime}$ is a signed $(k+1)$-dominating function for $G$, we have that

$$
\begin{aligned}
\delta_{A}(u) & =f^{\prime}\left(N_{A}(u)\right) \\
& =f^{\prime}(N[u])-f^{\prime}\left(N_{\bar{A}}(u)\right)-1 \\
& \geq k+1-f^{\prime}\left(N_{\bar{A}}(u)\right)-1 \\
& =\delta_{\bar{A}}(u)+k
\end{aligned}
$$

Thus, $A$ is a global defensive $k$-alliance in $G$.

Case 4: $u \in \bar{A}$. Since $A$ is a signed $(k+1)$-dominating function in $G$, we have that

$$
\begin{aligned}
\delta_{A}(u) & =f^{\prime}\left(N_{A}(u)\right) \\
& =f^{\prime}(N[u])-f^{\prime}\left(N_{\bar{A}}(u)\right)+1 \\
& \geq k+1-f^{\prime}\left(N_{\bar{A}}(u)\right)+1 \\
& =\delta_{\bar{A}}(u)+k+2
\end{aligned}
$$

Thus, $A$ is a global offensive $(k+2)$-alliance and, as a consequence, $A$ is a global powerful $k$-alliance in $G$. Therefore, the proof is complete.

Corollary 4. For any graph $G$ of order $n$ and any integer $k \in\{0, \ldots, \delta(G)\}$,

$$
\gamma_{s}^{k+1}(G)=2 \gamma_{k}^{p}(G)-n
$$

Proof: Let $S$ be a $\gamma_{k}^{p}(G)$-set. By Theorem 3, $f=\left(B_{1}=S, B_{-1}=\bar{S}\right)$ is a signed total $(k+1)$ dominating function of minimum weight in $G$. Thus $\gamma_{s}^{k+1}(G)=|S|-|\bar{S}|$. Since $|S|+|\bar{S}|=n$ and $\gamma_{k}^{p}(G)=|S|$, the result follows by adding these two equalities above.

According to the above ideas we can resume the relationships which motivated our work in the following table.

\begin{tabular}{|c|c|c|}
\hline$k$-monopoly $(k \geq 0)$ & $\Leftrightarrow$ & $\begin{array}{c}\text { Global defensive }(2 k) \text {-alliance and } \\
\text { global offensive }(2 k) \text {-alliance }\end{array}$ \\
\hline$k$-monopoly $(k \geq 1)$ & $\Leftrightarrow$ & Signed total $(2 k)$-domination \\
\hline Signed total $k$-domination $(k \geq 1)$ & $\Leftrightarrow$ & $\begin{array}{c}\text { Global defensive } k \text {-alliance and } \\
\text { global offensive } k \text {-alliance }\end{array}$ \\
\hline Signed $(k+1)$-domination $(k \geq 0)$ & $\Leftrightarrow$ & $\begin{array}{c}\text { Global defensive } k \text {-alliance and } \\
\text { global offensive }(k+2) \text {-alliance } \\
\text { (A global powerful } k \text {-alliance) }\end{array}$ \\
\hline
\end{tabular}

Notice that the definition of signed (total) $k$-dominating function is restricted to $k \geq 1$ while $k$-alliances are defined for any $k \in\{-\Delta(G), \ldots, \Delta(G)\}$ and $k$-monopolies can be defined for some integer $k$ whose limits are presented further. In this sense, these concepts being quite similar between them could be generalized for $k$ being zero or negative. To obtain a meaningful negative lower bound for $k$-monopolies 
we involve another well-known concept: total domination ${ }^{(\mathrm{i})}$. Namely, every $k$-monopoly, $k \geq 0$ is also a total dominating set for $G$. To remain this property also for $k<0$, we need to demand $\delta_{M}(v) \geq 1$ for every $v \in V(G)$. Therefore in this work we propose the following definition of monopolies and we study some of its mathematical properties.

Given a integer $k \in\left\{1-\left\lceil\frac{\delta(G)}{2}\right\rceil, \ldots,\left\lfloor\frac{\delta(G)}{2}\right\rfloor\right\}$ and a set $M$, a vertex $v$ of $G$ is said to be $k$-controlled by $M$ if $\delta_{M}(v) \geq \frac{\delta(v)}{2}+k$. The set $M$ is called a $k$-monopoly if it $k$-controls every vertex $v$ of $G$. The minimum cardinality of any $k$-monopoly is the $k$-monopoly number and it is denoted by $\mathcal{M}_{k}(G)$. A monopoly of cardinality $\mathcal{M}_{k}(G)$ is called a $\mathcal{M}_{k}(G)$-set. In particular notice that for a graph with a leaf (vertex of degree one), there exist only 0 -monopolies and the neighbor of every leaf is in each $\mathcal{M}_{0}$-set.

Notice that every non trivial graph $G$ contains at least one $k$-monopoly, with $k \in\left\{1-\left\lceil\frac{\delta(G)}{2}\right\rceil\right.$, $\left.\ldots,\left\lfloor\frac{\delta(G)}{2}\right\rfloor\right\}$, since every vertex of $G$ satisfies the $k$-monopoly condition for the whole vertex set $V(G)$. Also, if $G$ has an isolated vertex, $\mathcal{M}_{k}(G)$ does not exists. But if $G$ has no isolated vertices, then, since $\mathcal{M}_{k}(G)$-set is also a total dominating set, we have $\mathcal{M}_{k}(G) \geq 2$. Thus, we can say that in general for any graph $G$ of order $n, 2 \leq \mathcal{M}_{k}(G) \leq n$.

The last result of this section reveals a connection between $\mathcal{M}_{1-\left\lceil\frac{\delta(G)}{2}\right\rceil}(G)$ and $\gamma_{t}(G)$.

Theorem 5. For any $r$-regular graph $G$,

$$
\mathcal{M}_{1-\left\lceil\frac{r}{2}\right\rceil}(G)=\gamma_{t}(G) .
$$

Proof: Let $q=\frac{r}{2}+1-\left\lceil\frac{r}{2}\right\rceil$ and let $M$ be a $\mathcal{M}_{1-\left\lceil\frac{r}{2}\right\rceil}(G)$-set. If $r$ is even, then $q=1$ and if $r$ is odd, then $q=\frac{1}{2}$. In both cases, for any vertex $v$ of $G, \delta_{M}(v) \geq 1$, since $\delta_{M}(v)$ is an integer. Hence $M$ is a total dominating set and $\mathcal{M}_{1-\left\lceil\frac{r}{2}\right\rceil}(G) \geq \gamma_{t}(G)$. If $A$ is a $\gamma_{t}(G)$-set, then for every vertex $v \in V$ we obtain $\delta_{A}(v) \geq 1 \geq q$, since $q \in\left\{\frac{1}{2}, 1\right\}$. Thus, $A$ is also a $\left(1-\left\lceil\frac{r}{2}\right\rceil\right)$-monopoly and $\mathcal{M}_{1-\left\lceil\frac{r}{2}\right\rceil}(G) \leq \gamma_{t}(G)$, which yields the equality.

\section{Complexity}

Studies about complexity of signed domination were first presented by Hattingh et al. (1995). After that Henning (2004) has shown that signed total domination problem is NP-complete even restricted to bipartite or chordal graphs. This last work was continued by Liang (2014), where the NP-completeness of signed (total) $k$-domination problem was shown for $k \geq 2$. Consequently, by Theorem 1 the $k$-monopoly problem is also NP-complete for every $k \geq 1$. Hence, it remains to investigate the complexity of $k$ monopolies for $1-\left\lceil\frac{\delta(G)}{2}\right\rceil \leq k \leq 0$. As mentioned in the introduction, the complexity and also several inapproximation results are known for a closed monopolies, see Mishra (2012); Mishra and Rao (2006); Mishra et al. (2002); Peleg (2002).

On the other hand, also the global defensive $k$-alliance problem is NP-complete (unpublished manuscript Fernau (2013)) as well as global offensive $k$-alliance problem (see Fernau et al. (2009)), but not both together. Notice that global powerful $k$-alliance problem is NP-complete as shown by Fernau et al. (to

(i) A set $D$ is a total dominating set in a graph $G$ if every vertex of $G$ is adjacent to a vertex of $D$. The minimum cardinality of a total dominating set is the total domination number, denoted by $\gamma_{t}(G)$. 
appear) but, as we mention before, a global powerful $k$-alliance is a global defensive $k$-alliance and a global offensive $(k+2)$-alliance. Here we follow a similar approach as Hattingh et al. (1995) to show that 0 -monopoly problem is NP-complete. We will show the polynomial time reduction on the total domination set problem:

\section{Problem: TOTAL DOMINATION SET (TDS)}

INSTANCE: A graph $G$ and a positive integer $k \leq|V(G)|$.

QUESTION: Is $\gamma_{t}(G) \leq k$ ?

\section{Problem: 0-MONOPOLY}

INSTANCE: A graph $G$ and a positive integer $k \leq|V(G)|$.

QUESTION: Is $\mathcal{M}_{0}(G) \leq k$ ?

Recall that the total domination set problem is NP-complete even when restricted to bipartite graphs (see Laskar and Pfaff (1984)) or to chordal graphs (see Pfaff (1984)).

Theorem 6. Problem 0-MONOPOLY is NP-complete, even when restricted to bipartite or chordal graphs.

Proof: It is obvious that 0 -monopoly is a member of NP since for a given set $M$ with $|M| \leq k$ we can check in polynomial time for each vertex $v$ of a graph $G$ if $v$ is controlled by $M$.

Let $G$ be a graph of order $n$ and size $m$. We construct a graph $H$ from $G$ as follows. For every vertex $v$ add $\delta_{G}(v)-1$ paths on five vertices and connect $v$ with an edge to every middle vertex of these paths. Hence to obtain $H$ from $G$ we added $5 \sum_{v \in V(G)}\left(\delta_{G}(v)-1\right)=10 m-5 n$ vertices and the same amount of edges. (Notice that we have added exactly $4 m-2 n$ leaves.) Clearly this can be done in polynomial time. Also, if $G$ is bipartite or chordal graph, so is $H$. Next we claim $\mathcal{M}_{0}(H)=6 m-3 n+\gamma_{t}(G)$.

To prove this, let $M$ be a 0 -monopoly of $H$. Let $v_{1} v_{2} v_{3} v_{4} v_{5}$ be an arbitrary path added to $G$. Clearly $v_{2}, v_{4} \in M$, since they are unique neighbors of $v_{1}$ and $v_{5}$, respectively. Moreover, if $v_{3}$ is not in $M$, then both $v_{1}$ and $v_{5}$ must be in $M$ to control $v_{2}$ and $v_{4}$, respectively. Since $M$ has minimum cardinality, this implies that $v_{3} \in M$. Let $v \in V(G)$. By the above, $v$ has $\delta_{G}(v)-1$ neighbors in $M$ outside of $G$. Since $\delta_{H}(v)=2 \delta_{G}(v)-1, v$ needs an additional neighbor in $M \cap V(G)=P$ to be controlled by $M$. Hence, $P$ forms a total dominating set of $G$ and so $\gamma_{t}(G) \leq|P|$. Altogether

$$
\mathcal{M}_{0}(H)=|M|=|P|+3 \sum_{v \in V(G)}\left(\delta_{G}(v)-1\right) \geq \gamma_{t}(G)+6 m-3 n .
$$

On the other hand, suppose $S$ is a $\gamma_{t}(G)$-set of $G$. We will show that $M=S \cup\{v \in V(H)-V(G)$ : $\left.\delta_{H}(v)>1\right\}$ is a 0 -monopoly for $H$. Every vertex $v \in V(H)$ with $\delta_{H}(v)=1$ has a neighbor of degree two which is in $M$. Without loss of generality, every vertex $v \in V(H)-V(G)$ with $\delta_{H}(v)=2$ has one neighbor of degree 1 and the other neighbor which is in $M$ and we have $1=\delta_{M}(v) \geq \delta_{\bar{M}}(v)=1$. Every other vertex $v \in V(H)-V(G)$ has degree three, and two of its neighbors are in $V(H)-V(G)$ with degree two and thus they are in $M$. Hence $2=\delta_{M}(v) \geq \delta_{\bar{M}}(v)=1$. It remains to check vertices from $V(G)$. Let $v$ be a vertex with $\operatorname{deg}_{H}(v)=2 \operatorname{deg}_{G}(v)-1$. Since $S$ is a $\gamma_{t}(G)$ set, $v$ has at least one neighbor in $S$ and additional $\delta_{G}(v)-1$ vertices in $M$ in $V(H)-V(G)$. Altogether $v$ has at least 
$\delta_{G}(v)$ neighbors in $M$, which is more than half of its neighbors. The next calculation ends the proof of the claim:

$$
\begin{aligned}
\mathcal{M}_{0}(H) & \leq|M|=|S|+\left|\left\{v \in V(H)-V(G): \delta_{H}(v)>1\right\}\right| \\
& =\gamma_{t}(G)+3 \sum_{v \in V(G)}\left(\delta_{G}(v)-1\right) \\
& =\gamma_{t}(G)+6 m-3 n .
\end{aligned}
$$

Therefore, we have that if $j=6 m-3 n+k$, then $\gamma_{t}(G) \leq k$ if and only if $\mathcal{M}_{0}(H) \leq j$ and the proof is completed.

Once having studied the complexity of finding a 0-monopoly in a graph, it remains to investigate the complexity for $1-\left\lceil\frac{\delta(G)}{2}\right\rceil \leq k \leq-1$, which we leave as an open problem.

\section{Bounding $\mathcal{M}_{k}(G)$}

In this section we present bounds for $\mathcal{M}_{k}(G)$ with respect to the minimum and maximum degrees of $G$ and with respect to the order and size. First notice that the $k$-monopoly condition $\delta_{M}(v) \geq \frac{\delta(v)}{2}+k$ is equivalent to the following expressions:

$$
\delta_{\bar{M}}(v) \leq \frac{\delta(v)}{2}-k
$$

Theorem 7. Let $G$ be a graph of order $n$, minimum degree $\delta$ and maximum degree $\Delta$. Then for any integer $k \in\left\{1-\left\lceil\frac{\delta(G)}{2}\right\rceil, \ldots,\left\lfloor\frac{\delta(G)}{2}\right\rfloor\right\}$,

$$
\left\lceil\frac{\Delta+2 k+2}{2}\right\rceil \leq \mathcal{M}_{k}(G) \leq n-\left\lfloor\frac{\delta-2 k}{2}\right\rfloor \text {. }
$$

Proof: Let $A$ be a set of vertices of $G$ such that $|A|=\left\lfloor\frac{\delta-2 k}{2}\right\rfloor$ and let $v$ be a vertex of $G$. Hence $\delta_{A}(v) \leq\left\lfloor\frac{\delta-2 k}{2}\right\rfloor \leq \frac{\delta-2 k}{2}$. So,

$$
\delta_{\bar{A}}(v) \geq \delta(v)-\frac{\delta-2 k}{2} \geq \delta(v)-\frac{\delta(v)-2 k}{2}=\frac{\delta(v)+2 k}{2} .
$$

Thus we have $2 \delta_{\bar{A}}(v) \geq \delta(v)+2 k=\delta_{\bar{A}}(v)+\delta_{A}(v)+2 k$, which leads to $\delta_{\bar{A}}(v) \geq \delta_{A}(v)+2 k$. Therefore $\bar{A}$ is a $k$-monopoly in $G$ and the upper bound follows.

On the other hand, let $M$ be a $\mathcal{M}_{k}(G)$-set and let $u$ be a vertex of maximum degree in $G$. By (3) we have that

$$
\Delta=\delta_{M}(u)+\delta_{\bar{M}}(u) \leq \delta_{M}(u)+\frac{\delta(u)}{2}-k=\delta_{M}(u)+\frac{\Delta}{2}-k,
$$

which leads to $\frac{\Delta}{2}+k \leq \delta_{M}(u)$. Now, if $u \in M$, then we obtain that $\frac{\Delta}{2}+k \leq \delta_{M}(u) \leq|M|-1$ and, as a consequence, $\frac{\Delta+2 k+2}{2} \leq|M|$. Conversely, if $u \notin M$, then $\frac{\Delta}{2}+k \leq \delta_{M}(u) \leq|M|$ which leads to $\frac{\Delta+2 k}{2} \leq|M|$. Therefore, $|M| \geq \max \left\{\frac{\Delta+2 k}{2}, \frac{\Delta+2 k+2}{2}\right\}^{2}=\frac{\Delta+2 k+2}{2}$ and the lower bound follows.

As the following corollary shows the above bounds are tight. 
Corollary 8. For every complete graph $K_{n}$ and every $k \in\left\{1-\left\lceil\frac{\delta(G)}{2}\right\rceil, \ldots,\left\lfloor\frac{\delta(G)}{2}\right\rfloor\right\}$,

$$
\mathcal{M}_{k}\left(K_{n}\right)=\left\lceil\frac{n+2 k+1}{2}\right\rceil \text {. }
$$

Proof: From Theorem 7 we have that $\left\lceil\frac{n+2 k+1}{2}\right\rceil \leq \mathcal{M}_{k}\left(K_{n}\right) \leq n-\left\lfloor\frac{n-2 k-1}{2}\right\rfloor$. If $n-2 k-1$ is even, then $n+2 k+1$ is even and we obtain that

$$
\left\lceil\frac{n+2 k+1}{2}\right\rceil \leq \mathcal{M}_{k}\left(K_{n}\right) \leq n-\left\lfloor\frac{n-2 k-1}{2}\right\rfloor=n-\frac{n-2 k-1}{2}=\frac{n+2 k+1}{2}=\left\lceil\frac{n+2 k+1}{2}\right\rceil .
$$

On the other hand, if $n-2 k-1$ is odd, then $n+2 k+1$ is odd and we have that

$$
\left\lceil\frac{n+2 k+1}{2}\right\rceil \leq \mathcal{M}_{k}\left(K_{n}\right) \leq n-\left\lfloor\frac{n-2 k-1}{2}\right\rfloor=n-\frac{n-2 k-2}{2}=\frac{n+2 k+2}{2}=\left\lceil\frac{n+2 k+1}{2}\right\rceil \text {. }
$$

Next we obtain a lower bound for $\mathcal{M}_{k}(G)$ in terms of order and size of $G$.

Theorem 9. For any graph $G$ of order $n$ and size $m$ and for every $k \in\left\{1-\left\lceil\frac{\delta(G)}{2}\right\rceil, \ldots,\left\lfloor\frac{\delta(G)}{2}\right\rfloor\right\}-\{0\}$,

$$
\mathcal{M}_{k}(G) \geq\left\lceil\frac{3 k n-m}{2 k}\right\rceil .
$$

Proof: Let $M$ be a $\mathcal{M}_{k}(G)$-set. Since every vertex $v \in \bar{M}$ satisfies that $\delta_{M}(v) \geq \delta_{\bar{M}}(v)+2 k \geq 2 k$, we have that $c(M, \bar{M}) \geq 2 k|\bar{M}|=2 k(n-|M|)$, where $c(M, \bar{M})$ is the edge cut set between $M$ and $\bar{M}$. Since $\delta_{M}(v) \geq \delta_{\bar{M}}(v)+2 k$ holds for every vertex $v \in M$, we have

$$
\begin{aligned}
2 k|\bar{M}| & \leq c(M, \bar{M}) \\
& =\sum_{v \in M} \delta_{\bar{M}}(v) \\
& \leq \sum_{v \in M}\left(\delta_{M}(v)-2 k\right) \\
& =2|E(\langle M\rangle)|-2 k|M|,
\end{aligned}
$$

which leads to $|E(\langle M\rangle)| \geq k n$. Since $m \geq|E(\langle M\rangle)|+c(M, \bar{M})$, we obtain that $m \geq k n+2 k(n-$ $|M|)=3 k n-2 k|M|$ and the result follows.

To see the tightness of the above bound we consider the following family $\mathcal{F}$ of graphs. We begin with a complete graph $K_{t}$ with set of vertices $V=\left\{v_{0}, v_{1}, \ldots, v_{t-1}\right\}$ and $t-1 \equiv 0(\bmod 4)$ and $t$ isolated vertices $U=\left\{u_{0}, u_{1}, \ldots, u_{t-1}\right\}$. From now on all the operations with subindexes of $v_{i}$ or $u_{i}$ are done modulo $t$. To obtain a graph $G \in \mathcal{F}$, for every $i \in\{0, \ldots, t-1\}$, we add the edges $u_{i} v_{i}, u_{i} v_{i+1}$, $u_{i} v_{i+2}, \ldots, u_{i} v_{i+(t-3) / 2}$. Notice that $G$ has order $2 t$ and size $t(t-1)$ and every vertex $v_{i} \in V$ has $\frac{t-1}{2}$ neighbors in $U$ and vice versa. Hence $\delta(G)=\frac{t-1}{2}$. Suppose $k=\left\lfloor\frac{t-1}{4}\right\rfloor$. If $v \in V$, then $\delta_{V}(v)=t-1=$ $\frac{t-1}{2}+\frac{t-1}{2}=\delta_{U}(v)+\frac{t-1}{2}=\delta_{U}(v)+2 k$. Also if $v \in U$, then $\delta_{V}(v) \stackrel{4}{=} \frac{t-1}{2}=\delta_{U}(v)+\frac{t-1}{2}=\delta_{U}(v)+2 k$. Thus $V$ is a $k$-monopoly in $G$. By Theorem 9 we have $\mathcal{M}_{k}(G)=t$ and the bound is achieved. 
Theorem 10. For any r-regular graph $G$ of order $n$ and for every $k \in\left\{1-\left\lceil\frac{\delta(G)}{2}\right\rceil, \ldots,\left\lfloor\frac{\delta(G)}{2}\right\rfloor\right\}$,

$$
\mathcal{M}_{k}(G) \geq\left\lceil\frac{n(2 k+r)}{2 r}\right\rceil \text {. }
$$

Proof: Let $V$ be the vertex set of $G$ and let $M$ be a $\mathcal{M}_{k}(G)$-set. For any vertex $v \in V$ and any $M \subset V$ we have that $\delta(v)=\delta_{M}(v)+\delta_{\bar{M}}(v)$. By subtracting $2 \delta_{\bar{M}}(v)$ in both sides of the equality we obtain $\delta(v)-2 \delta_{\bar{M}}(v)=\delta_{M}(v)-\delta_{\bar{M}}(v)$. Making a sum for every vertex of $G$ and using the fact that $G$ is $r$-regular, it follows

$$
\sum_{v \in V}\left(\delta_{M}(v)-\delta_{\bar{M}}(v)\right)=\sum_{v \in V}\left(\delta(v)-2 \delta_{\bar{M}}(v)\right)=n r-2 \sum_{v \in V} \delta_{\bar{M}}(v)=n r-2 r|\bar{M}|=r|M|-r|\bar{M}| .
$$

Thus, $\sum_{v \in V}\left(\delta_{M}(v)-\delta_{\bar{M}}(v)\right)=r|M|-r|\bar{M}|$. Since every vertex $v \in V$ satisfies $\delta_{M}(v) \geq \delta_{\bar{M}}(v)+2 k$, we have

$$
2 k n=\sum_{v \in V} 2 k \leq \sum_{v \in V}\left(\delta_{M}(v)-\delta_{\bar{M}}(v)\right)=r|M|-r|\bar{M}|=2 r|M|-r n
$$

and the result follows.

As we will see in Proposition 15, the above bound is tight. For instance, it is achieved for the case of cycles $C_{4 t}$ for $k=0$.

\section{Exact values for $\mathcal{M}_{k}(G)$}

As already mentioned, for any graph $G$ of order $n, 2 \leq \mathcal{M}_{k}(G) \leq n$. We first characterize the classes of graphs achieving the limit cases for these bounds.

Proposition 11. Let $G$ be a graph of order $n$. Then $\mathcal{M}_{k}(G)=2$ if and only if $G$ is isomorphic to $P_{2}, P_{3}$, $P_{4}, C_{3}$ or $C_{4}$. Moreover, $k$ is either 0 or 1 .

Proof: If $G$ is isomorphic to $P_{2}, P_{3}, P_{4}, C_{3}$ or $C_{4}$, then $\delta(G) \leq 2, k \in\{0,1\}$ and $\mathcal{M}_{k}(G)=2$. On the contrary, suppose that $\mathcal{M}_{k}(G)=2$. Let $S=\{u, v\}$ be a $\mathcal{M}_{k}(G)$-set. Notice that $u$ and $v$ must be adjacent. So, $\delta_{\bar{S}}(u) \leq 1$ and $\delta_{\bar{S}}(v) \leq 1$ and $G$ must contain at most four vertices. Moreover, for every vertex $x \notin\{u, v\}$ it follows $\delta_{\bar{S}}(x) \leq 1$. Thus, $\delta(G) \leq 2, k \in\{0,1\}$ and we have the following cases. If $\delta_{\bar{S}}(u)=0$ and $\delta_{\bar{S}}(v)=0$, then $G$ is isomorphic to $P_{2}$. If $\delta_{\bar{S}}(u)=1$ and $\delta_{\bar{S}}(v)=0$ (or vice versa), then $G$ is isomorphic to $P_{3}$. If $\delta_{\bar{S}}(u)=1$ and $\delta_{\bar{S}}(v)=1$, then $G$ is isomorphic either to $P_{4}, C_{3}$ or $C_{4}$, which completes the proof.

Proposition 12. Let $G$ be a graph of order $n$ and minimum degree $\delta$. Then $\mathcal{M}_{k}(G)=n$ if and only if $k=\left\lfloor\frac{\delta}{2}\right\rfloor$ and either

(i) $\delta$ is even and every vertex of $G$ is adjacent to a vertex of degree $\delta$ or $\delta+1$, or

(ii) $\delta$ is odd and every vertex of $G$ is adjacent to a vertex of degree $\delta$. 
Proof: Suppose $\mathcal{M}_{k}(G)=n$. Hence, for any vertex $v \in V(G), M=V(G)-\{v\}$ is not $k$-monopoly in $G$. Thus the vertex $v$ or some vertex $u \in N(v)$ does not satisfy the monopoly condition. If $\delta_{M}(v)<$ $\delta_{\bar{M}}(v)+2 k$, then we have that $\delta(v)<2 k \leq \delta$, a contradiction. Thus $\delta_{M}(u)<\delta_{\bar{M}}(u)+2 k$, which leads to $\delta(u)-1<1+2 k$. So $\delta(u) \leq 2 k+1$. As a consequence, we obtain that $k \geq \frac{\delta-1}{2}$ (or equivalently $k \geq\left\lceil\frac{\delta-1}{2}\right\rceil$ ). Since $k \leq\left\lfloor\frac{\delta}{2}\right\rfloor$, we obtain that $k=\left\lfloor\frac{\delta}{2}\right\rfloor=\left\lceil\frac{\delta-1}{2}\right\rceil$. Thus, $\delta(u) \leq 2 k+1=2\left\lceil\frac{\delta-1}{2}\right\rceil+1$. Hence, if $\delta$ is even, then we have that $\delta(u) \leq \delta+1$, and if $\delta$ is odd, then we have that $\delta(u) \leq \delta$. Therefore, (i) and (ii) follow.

On the other hand, suppose $k=\left\lfloor\frac{\delta}{2}\right\rfloor$. Assume $\delta$ is even and every vertex of $G$ is adjacent to a vertex of degree $\delta$ or $\delta+1$. Hence, let $M \subset V(G)$, let $x \notin M$ and let $u \in N(x)$ having degree $\delta$ or $\delta+1$. So we have,

$$
\delta_{M}(u) \leq \delta<2\left\lfloor\frac{\delta}{2}\right\rfloor+1=2 k+1 \leq \delta_{\bar{M}}(u)+2 k .
$$

Thus, $M$ is not a $k$-monopoly.

Now, suppose $\delta$ is odd and every vertex of $G$ is adjacent to a vertex of degree $\delta$. As above let $M^{\prime} \subset$ $V(G)$, let $x^{\prime} \notin M^{\prime}$ and let $u^{\prime} \in N\left(x^{\prime}\right)$ having degree $\delta$. So we have,

$$
\delta_{M^{\prime}}\left(u^{\prime}\right)<\delta=2\left\lfloor\frac{\delta}{2}\right\rfloor+1=2 k+1 \leq \delta_{\overline{M^{\prime}}}\left(u^{\prime}\right)+2 k .
$$

Thus, $M^{\prime}$ is not a $k$-monopoly.

Therefore, any proper subset of $V(G)$ is not a $k$-monopoly and we have that $\mathcal{M}_{k}(G)=n$.

The wheel graph of order $n$ is defined as $W_{1, n-1}=K_{1}+C_{n-1}$, where + represents the join of mentioned graphs. The fan graph $F_{1, n-1}$ of order $n$ is defined as the graph $K_{1}+P_{n-1}$.

\section{Corollary 13.}

(i) For any r-regular graph $G$ of order $n, \mathcal{M}_{\left\lfloor\frac{r}{2}\right\rfloor}(G)=n$.

(ii) For any wheel graph $W_{1, n-1}, \mathcal{M}_{1}\left(W_{1, n-1}\right)=n$.

(iii) For any fan graph $F_{1, n-1}, \mathcal{M}_{1}\left(F_{1, n-1}\right)=n$.

(iv) For any bipartite graph $K_{r, r+1}$, r even, $\mathcal{M}_{\left\lfloor\frac{r}{2}\right\rfloor}\left(K_{r, r+1}\right)=2 r+1$.

We continue this section by obtaining exact values for some graph classes. Recall that, by Corollary 8 , for $k \in\left\{1-\left\lceil\frac{\delta(G)}{2}\right\rceil, \ldots,\left\lfloor\frac{\delta(G)}{2}\right\rfloor\right\}$ we have $\mathcal{M}_{k}\left(K_{n}\right)=\left\lceil\frac{n+2 k+1}{2}\right\rceil$. We continue with complete bipartite graphs.

Proposition 14. For every complete bipartite graph $K_{r, t}$ and every $k \in\left\{1-\left\lceil\frac{\delta(G)}{2}\right\rceil, \ldots,\left\lfloor\frac{\delta(G)}{2}\right\rfloor\right\}$,

$$
\mathcal{M}_{k}\left(K_{r, t}\right)=\left\lceil\frac{r+2 k}{2}\right\rceil+\left\lceil\frac{t+2 k}{2}\right\rceil .
$$


Proof: Let $X$ and $Y$ be the partition sets of $K_{r, t}$ such that $|X|=r$ and $|Y|=t$ and let $S$ be a subset of vertices of $K_{r, t}$ such that $|S \cap X|=\left\lceil\frac{r+2 k}{2}\right\rceil$ and $|S \cap Y|=\left\lceil\frac{t+2 k}{2}\right\rceil$. Let $v$ be a vertex of $K_{r, t}$. If $v \in X$, then

$$
\delta_{S}(v)=\left\lceil\frac{t+2 k}{2}\right\rceil \geq \frac{t+2 k}{2}=t+2 k-\frac{t+2 k}{2} \geq t-\left\lceil\frac{t+2 k}{2}\right\rceil+2 k=\delta_{\bar{S}}(v)+2 k .
$$

Analogously, if $v \in Y$, then we obtain that $\delta_{S}(v) \geq \delta_{\bar{S}}(v)+2 k$. Thus, $S$ is a $k$-monopoly in $K_{r, t}$ and we have that $\mathcal{M}_{k}\left(K_{r, t}\right) \leq\left\lceil\frac{r+2 k}{2}\right\rceil+\left\lceil\frac{t+2 k}{2}\right\rceil$.

Now, let $M$ be a $\mathcal{M}_{k}\left(K_{r, t}\right)$-set and let $u$ be a vertex of $K_{r, t}$. If $u \in X$, then we have that $\delta_{M}(u) \geq$ $\delta_{\bar{M}}(u)+2 k=t-\delta_{M}(u)+2 k$, which leads to $\delta_{M}(u) \geq \frac{t+2 k}{2}$ and, as a consequence, $|Y \cap M|=$ $\delta_{M}(u) \geq\left\lceil\frac{t+2 k}{2}\right\rceil$. Analogously, if $u \in Y$, then we obtain that $|X \cap M| \geq\left\lceil\frac{r+2 k}{2}\right\rceil$. Thus, $\mathcal{M}_{k}\left(K_{r, t}\right)=$ $|M \cap X|+|M \cap Y| \geq\left\lceil\frac{r+2 k}{2}\right\rceil+\left\lceil\frac{t+2 k}{2}\right\rceil$ and the proof is complete.

Next we study $k$-monopolies of cycles and paths. First notice that the case $k=1$ for cycles follows directly from Corollary 13 (i), that is, $\mathcal{M}_{1}\left(C_{n}\right)=n$.

Proposition 15. For every integer $n \geq 3$,

$$
\mathcal{M}_{0}\left(C_{n}\right)=\mathcal{M}_{0}\left(P_{n}\right)= \begin{cases}\frac{n}{2} & \text { if } n \equiv 0 \bmod 4 \\ \frac{n+2}{2} & \text { if } n \equiv 2 \bmod 4 \\ \frac{n+1}{2} & \text { if } n \equiv 1 \bmod 4 \operatorname{or} n \equiv 3 \bmod 4 .\end{cases}
$$

Proof: By Theorem 5, $\mathcal{M}_{0}\left(C_{n}\right)=\gamma_{t}\left(C_{n}\right)$ and it is known from Henning (2000) that $\gamma_{t}\left(C_{n}\right)=\left\lfloor\frac{n}{2}\right\rfloor+$ $\left\lceil\frac{n}{4}\right\rceil-\left\lfloor\frac{n}{4}\right\rfloor$. Hence we are done with cycles.

Let $V\left(P_{n}\right)=\left\{v_{0}, \ldots, v_{n-1}\right\}$. We proceed by induction on $k \geq 1$ where $n=4 k+i$ and $i \in$ $\{-1,0,1,2\}$. Let $M_{n}$ be a subset of $V\left(P_{n}\right)$ defined as follows.

- If $n \equiv 0(\bmod 4)$, then $M_{n}=\left\{v_{1}, v_{2}, v_{5}, v_{6}, \ldots, v_{n-3}, v_{n-2}\right\}$.

- If $n \equiv 1(\bmod 4)$, then $M_{n}=\left\{v_{1}, v_{2}, v_{3}, v_{6}, v_{7}, v_{10}, v_{11}, \ldots, v_{n-3}, v_{n-2}\right\}$.

- If $n \equiv 2(\bmod 4)$, then $M_{n}=\left\{v_{0}, v_{1}, v_{3}, v_{4}, v_{7}, v_{8}, v_{11}, v_{12}, \ldots, v_{n-3}, v_{n-2}\right\}$.

- If $n \equiv 3(\bmod 4)$, then $M_{n}=\left\{v_{0}, v_{1}, v_{4}, v_{5}, \ldots, v_{n-3}, v_{n-2}\right\}$.

It is straightforward to check that $M_{n}$ is a $\mathcal{M}_{0}\left(P_{n}\right)$-set for $k=1$. Notice that $M_{4}$ is the unique $\mathcal{M}_{0}\left(P_{4}\right)$-set. Let $k>1$. Set $M_{4(k-1)+i}$ is a $\mathcal{M}_{0}\left(P_{4(k-1)+i}\right)$-set by induction hypothesis. Clearly, any 0 -monopoly $M^{\prime}$ of $P_{n}$ contains at least two vertices of the last three vertices $v_{n-3}, v_{n-2}, v_{n-1}$. Hence, these two vertices have no influence on the vertices of $M^{\prime}$ from the first $4(k-1)+i$ vertices of the path $P_{4 k+i}$. Therefore $\left|M^{\prime} \cap\left\{v_{0}, \ldots, v_{4(k-1)+i}\right\}\right| \geq\left|M_{4(k-1)+i}\right|$ and $M_{4 k+i}=M_{4(k-1)+i} \cup\left\{v_{n-3}, v_{n-2}\right\}$ is a $\mathcal{M}_{0}\left(P_{4 k+i}\right)$-set. It is easy to see that $\left|M_{4 k+i}\right|$ gives the desired values. 


\section{Partitions into $k$-monopolies}

In this section we present some results about partitioning a graphs into monopolies. To this end, we say that a graph $G=(V, E)$ is $k$-monopoly partitionable if there exists a vertex partition $\Pi=\left\{S_{1}, \ldots, S_{r}\right\}$ of $V, r \geq 2$, such that for every $i \in\{1, \ldots, r\}, S_{i}$ is a $k$-monopoly in $G$.

Theorem 16. If a graph $G$ is $k$-monopoly partitionable, for some $k \in\left\{1-\left\lceil\frac{\delta(G)}{2}\right\rceil, \ldots,\left\lfloor\frac{\delta(G)}{2}\right\rfloor\right\}$, then $r \leq 2-2 k$ and $k \leq 0$.

Proof: Let $S_{i}, S_{j} \in \Pi$ and let $v$ be a vertex of $G$. Then we have that

$$
\begin{aligned}
\delta_{S_{i}}(v) & \geq \delta_{\overline{S_{i}}}(v)+2 k \\
& =2 k+\sum_{\ell=1, \ell \neq i}^{r} \delta_{S_{\ell}}(v) \\
& =\delta_{S_{j}}(v)+2 k+\sum_{\ell=1, \ell \neq i, j}^{r} \delta_{S_{\ell}}(v) \\
& \geq \delta_{\overline{S_{j}}}(v)+4 k+\sum_{\ell=1, \ell \neq i, j}^{r} \delta_{S_{\ell}}(v)
\end{aligned}
$$

Since for every $u$ of $G, \delta_{S_{\ell}}(u) \geq 1$ for every $\ell \in\{1, \ldots, r\}$, we obtain that $\sum_{\ell=1, \ell \neq i, j}^{r} \delta_{S_{\ell}}(u) \geq r-2$. So,

$$
\begin{aligned}
\delta_{S_{i}}(v) & \geq \delta_{\overline{S_{j}}}(v)+4 k+r-2 \\
& =4 k+r-2+\sum_{\ell=1, \ell \neq j}^{r} \delta_{S_{\ell}}(v) \\
& =\delta_{S_{i}}(v)+4 k+r-2+\sum_{\ell=1, \ell \neq i, j}^{r} \delta_{S_{\ell}}(v) \\
& \geq \delta_{S_{i}}(v)+4 k+2 r-4 .
\end{aligned}
$$

Thus $2 k+r-2 \leq 0$, which leads to $r \leq 2-2 k$ and $k \leq 1-r / 2$. Since $r \geq 2$, we have that $k \leq 0$.

From the above result we have that $G$ can be only partitioned into at most $2-2 k k$-monopolies for $k \leq 0$. The particular case $k=0$ is next studied. Notice that for instance, cycles of order $4 t$ and hypercubes $Q_{2 t}$ with $t \geq 1$ are examples of graphs having a partition into two 0 -monopolies.

Proposition 17. Let $G$ be a graph having a vertex partition into two 0-monopolies $\{X, Y\}$. Then the following assertions are satisfied.

(i) For every vertex $v$ of $G, \delta_{X}(v)=\delta_{Y}(v)$.

(ii) For every vertex $v$ of $G, \delta(v)$ is an even number. 
(iii) The size $m_{X}$ of $\langle X\rangle$ equals the size $m_{Y}$ of $\langle Y\rangle$.

(iv) The cardinality of the edge cut set $c(X, Y)$ produced by the vertex partition $\{X, Y\}$ equals the size $m$ of $G$ minus two times the size of $\langle X\rangle$.

Proof: For every vertex $v$ of $G$ we have that $\delta_{X}(v) \geq \delta_{Y}(v)$ and $\delta_{Y}(v) \geq \delta_{X}(v)$. Thus, (i) follows. Now, (ii) follows from the fact that $\delta(v)=\delta_{X}(v)+\delta_{Y}(v)=2 \delta_{X}(v)=2 \delta_{Y}(v)$. To prove (iii) we consider the following

$$
\sum_{v \in X} \delta_{X}(v)+\sum_{v \in Y} \delta_{X}(v)=\sum_{v \in X} \delta_{Y}(v)+\sum_{v \in Y} \delta_{Y}(v)
$$

Since, $\sum_{v \in Y} \delta_{X}(v)=\sum_{v \in X} \delta_{Y}(v)$ we have the result. As a consequence, $m=c(X, Y)+m_{X}+m_{Y}$ and by (iii) we obtain (iv).

A natural question which now arises concerning the computational complexity on the existence of such partitions mentioned above. That is for instance, given a graph $G$, can we decide whether $G$ is $k$ monopoly partitionable? Moreover, if the answer is positive, can we find such partitions by using some efficient algorithm?

\section{References}

J.-C. Bermond, J. Bond, D. Peleg, and S. Perennes. The power of small coalitions in graphs. Discrete Applied Mathematics, 127(3):399-414, 2003. doi: 10.1016/S0166-218X(02)00241-X. URL http: //www.scopus.com/inward/record.url ?eid=2-s2.0-84867959143\&partnerID= $40 \& m d 5=d f 993 b d d b d f 24 f 26 b 0 f 2554323 b c e 429$.

J. Dunbar, S. Hedetniemi, M. Henning, and P. Slater. Signed domination in graphs. Graph Theory, Combinatorics, and Applications, pages 311-322, 1995.

C. Dwork, D. Peleg, N. Pippenger, and E. Upfal. Fault tolerance in networks of bounded degree. SIAM Journal on Computing, 17(5):975-988, 1988. URL http://www.scopus. com/inward/record.url?eid=2-s2.0-0024091299\&partnerID $=40$ \&m5 = $009 \mathrm{c} 11 \mathrm{dd} 0 \mathrm{~d} 050861 \mathrm{~b} 69499 \mathrm{~d} 3 \mathrm{fbf} 93566$.

O. Favaron, G. Fricke, W. Goddard, S. Hedetniemi, S. Hedetniemi, P. Kristiansen, R. C. Laskar, and R. Skaggs. Offensive alliances in graphs. Discussiones Mathematicae Graph Theory, 24:263-275, 2004.

H. Fernau. Private communication. 2013.

H. Fernau and J. Rodríguez-Velázquez. A survey on alliances and related parameters in graphs. Electronic Journal of Graph Theory and Applications, 2(1):70-86, 2014.

H. Fernau, J. Rodríguez-Velázquez, and J. Sigarreta. Offensive r-alliances in graphs. Discrete Applied Mathematics, 157(1):177-182, 2009. doi: 10.1016/j.dam.2008.06.001. URL http: //www. scopus.com/inward/record.url?eid=2-s2.0-56649115970\&partnerID= $40 \& \mathrm{md} 5=\mathrm{ec} 6162 \mathrm{e} 86 \mathrm{~b} 28 \mathrm{fe} 3 \mathrm{af} 48 \mathrm{f} 2 \mathrm{f} 1 \mathrm{c} 64169 \mathrm{a} 75$. 
H. Fernau, J. Rodríguez-Velázquez, and J. Sigarreta. Global powerful r-alliances and total k-domination in graphs. Utilitas Mathematica, to appear.

P. Flocchini, R. Královič, P. Ružička, A. Roncato, and N. Santoro. On time versus size for monotone dynamic monopolies in regular topologies. Journal of Discrete Algorithms, 1(2): 129-150, 2003. doi: 10.1016/S1570-8667(03)00022-4. URL http://www.scopus . $\mathrm{com} / \mathrm{inward} / \mathrm{record}$.url ?eid=2-s2 $.0-77952569578 \&$ partner $\mathrm{ID}=40 \& \mathrm{md} 5=$ bc3c283a056a43366c34cdffad9c85e3.

H. Garcia-Molina and D. Barbara. How to assign votes in a distributed system. Journal of the ACM, 32(4):841-860, 1985 . doi: 10.1145/4221.4223. URL http://www.scopus. $\mathrm{com} /$ inward/record.url?eid=2-s2.0-0022145769\&partnerID $=40$ \& md5 = $19 a 2 c a 07 b 3 b 1 f 38508 e 2926817239 a c d$.

J. Hattingh, M. Henning, and P. Slater. The algorithmic complexity of signed domination in graphs. Australasian Journal of Combinatorics, 12:101-112, 1995.

T. Haynes, S. Hedetniemi, and M. Henning. Global defensive alliances in graphs. Electronic Journal of Combinatorics, 10(1 R), 2003. URL http: / /www. scopus.com/inward/record.url?eid= $2-\mathrm{s} 2.0-3042619803 \&$ partner ID $=40$ \& md5 =de $525879814 \mathrm{c} 4 \mathrm{abb} f 82 \mathrm{~b} 4 \mathrm{~b} 7 \mathrm{~d} 27 \mathrm{f} 52 \mathrm{fa} 4$.

M. Henning. Graphs with large total domination number. Journal of Graph Theory, 35 (1):21-45, 2000. URL http://www.scopus.com/inward/record.url?eid=2-s2 . $0-23044518657 \&$ partner ID $=40$ \&md5=eda 9769 f $06132678 \mathrm{c} 59 \mathrm{dd} 3470 \mathrm{~b} 350 \mathrm{fe} 7$.

M. Henning. Signed total domination in graphs. Discrete Mathematics, 278(1-3):109-125, 2004. doi: 10.1016/j.disc.2003.06.002. URL http://www.scopus.com/inward/record.url?eid= $2-s 2.0-1142263889 \&$ part ner ID $=40$ \& md5 $=20$ dd 482 bbe $17 \mathrm{~d} 6173990 \mathrm{~b} 948169 \mathrm{f} 1 \mathrm{bbe}$.

K. Khoshkhah, M. Nemati, H. Soltani, and M. Zaker. A study of monopolies in graphs. Graphs and Combinatorics, 29(5):1417-1427, 2013. doi: 10.1007/s00373-012-1214-7. URL http: //www.scopus.com/inward/record.url?eid=2-s2 .0-84882903032\&partnerID= 40 \&md5 $=9962 \mathrm{cc} 0$ de $8828815 \mathrm{ac} 6749 \mathrm{~b} 93652024 \mathrm{~d}$.

P. Kristiansen, S. Hedetniemi, and S. Hedetniemi. Alliances in graphs. Journal of Combinatorial Mathematics and Combinatorial Computing, 48:157-177, 2004.

R. Laskar and J. Pfaff. Domination and irredundance in split graphs. Technical Report, Department of Mathematical Sciences, Clemson University, 10(1):49-60, 1984. doi: 10.1016/ j.jda.2011.12.019. URL http://www.scopus.com/inward/record.url?eid=2-s2. $0-84855548350$ \&partnerID $=40$ \&md5 $=468858 \mathrm{cae} 7328 \mathrm{c} 688 \mathrm{f} 73 \mathrm{~b} 0 \mathrm{e} 203 \mathrm{~b} 00 \mathrm{ac} 6$.

H. Liang. On the signed (total) $k$-domination number of a graph. Journal of Combinatorial Mathematics and Combinatorial Computing, 89:87-99, 2014.

N. Linial, D. Peleg, Y. Rabinovich, and M. Saks. Sphere packing and local majorities in graphs. $2^{\text {nd }}$ Israel Symposium on Theory and Computing Systems, pages 141-149, 1993. 
S. Mishra. Complexity of majority monopoly and signed domination problems. Journal of Discrete Algorithms, 10(1):49-60, 2012. doi: 10.1016/j.jda.2011.12.019. URL http: //www. scopus.com/inward/record. url ?eid=2-s2.0-84855548350\&partnerID= $40 \& \mathrm{md} 5=468858 \mathrm{cae} 7328 \mathrm{c} 688 \mathrm{f} 73 \mathrm{~b} 0 \mathrm{e} 203 \mathrm{~b} 00 \mathrm{ac} 6$.

S. Mishra and S. Rao. Minimum monopoly in regular and tree graphs. Discrete Mathematics, 306(14):1586-1594, 2006. doi: 10.1016/j.disc.2005.06.036. URL http: //www. scopus.com/inward/record. url ?eid=2-s2.0-33745656404\&partnerID= $40 \& \mathrm{md} 5=0 \mathrm{f} 2 \mathrm{c} 2 \mathrm{e} 220120 \mathrm{fbe} 991066315 \mathrm{ad} 65 \mathrm{cdc} 6$.

S. Mishra, J. Radhakrishnan, and S. Sivasubramanian. On the hardness of approximating minimum monopoly problems. Lecture Notes in Computer Science (including subseries Lecture Notes in Artificial Intelligence and Lecture Notes in Bioinformatics), 2556 LNCS: 277-288, 2002. URL http://www.scopus.com/inward/record.url?eid=2-s2. $0-80052395587 \&$ partner ID $=40$ \&md5 =cb2 4 b2 a db $61 \mathrm{a} 5 \mathrm{db} f f 36910 \mathrm{~d} 9 \mathrm{addb} a 94 \mathrm{c}$.

D. Peleg. Local majorities, coalitions and monopolies in graphs: A review. Theoretical Computer Science, 282(2):231-257, 2002. doi: 10.1016/S0304-3975(01)00055-X. URL http: //www.scopus.com/inward/record.url?eid=2-s2.0-0037054318\&partnerID= $40 \& \mathrm{md} 5=32 \mathrm{~b} 9 \mathrm{c} 2596729$ f62832b1564d367adccc.

J. Pfaff. Algorithmic complexities of domination-related graph parameters. Ph.D. Thesis, Clemson University, 1984.

K. Shafique and R. Dutton. Maximum alliance-free and minimum alliance-cover sets. Congressus Numerantium, 162:139-146, 2003.

K. Shafique and R. Dutton. A tight bound on the cardinalities of maximun alliance-free and minimun alliance-cover sets. Journal of Combinatorial Mathematics and Combinatorial Computing, 56:139$145,2006$.

G. Sullivan. The complexity of system-level fault diagnosis and diagnosability. Ph. D. Thesis, Yale University New Haven. CT, 1986.

M. Zaker. On dynamic monopolies of graphs with general thresholds. Discrete Mathematics, 312(6):1136-1143, 2012. doi: 10.1016/j.disc.2011.11.038. URL http://www.scopus . $\mathrm{com} /$ inward/record.url ?eid=2-s2.0-84155191040\&partner ID $=40$ \&m5 $=$ c463fb9151e8e07ebad6dc45643a750e.

B. Zelinka. Signed total domination number of a graph. Czechoslovak Mathematical Journal, 51 (2):225-229, 2001. URL http://www.scopus.com/inward/record.url?eid=2-s2. $0-23044528767 \&$ partner ID $=40$ \&md5=9024d6b $751 \mathrm{af} 95 \mathrm{~d} 67 \mathrm{c} 4254 \mathrm{a} 2 \mathrm{~d} 6 \mathrm{a} 091 \mathrm{e} 6$. 